\title{
Hypoglycemic property of soy isoflavones from hypocotyl in Goto-Kakizaki diabetic rats
}

\author{
Ming Jin,,$^{1,+}$ Ming-hua Shen, ${ }^{1,+}$ Mei-hua Jin, ${ }^{1}$ Ai-hua Jin, ${ }^{2}$ Xue-zhe Yin ${ }^{2, *}$ and Ji-shu Quan ${ }^{1, *}$ \\ 'Department of Biochemistry and Molecular Biology, Yanbian University Medical College, Yanji 133002, China \\ ${ }^{2}$ Affiliated Hospital of Yanbian University, Yanji 133000, China
}

(Received 16 July, 2017; Accepted 28 August, 2017; Published online 12 December, 2017)

\begin{abstract}
The present study was carried out to investigate the hypoglycemic effect of soy isoflavones from hypocotyl in GK diabetic rats. A single administration and long-term administration tests were conducted in GK diabetic rats to test the hypoglycemic effect of soy isoflavones. At the end of long-term administration trial, blood protein, cholesterol, triglyceride, glycosylated serum protein, C-reactive protein, insulin, aminotransferase, lipid peroxide, interleukin-6, tumor necrosis factor- $\alpha$ were estimated. Inhibition of soy isoflavones against $\alpha$-amylase and $\alpha$-glucosidase, as well as on glucose uptake into brush border membrane vesicles or Caco-2 cells were determined in vitro. In single administration experiment, soy isoflavones reduced postprandial blood glucose levels in GK rats. In long-term administration, hypoglycemic effect of soy isoflavones was first observed at week 12 and maintained till week 16 . A significant reduction in fasting blood glucose, C-reactive protein, and lipid peroxide was noted at week 16 . However, there was no significant treatment effect on blood insulin. Furthermore, soy isoflavone administration resulted in significant decreases in glycosylated serum protein, tumor necrosis factor- $\alpha$, and interleukin- 6 . Other biochemical parameters, such as protein, cholesterol, triglyceride and aminotransferases were not modified, however. The results in vitro showed that soy isoflavones showed a potent inhibitory effect on intestinal $\alpha$-glucosidase, but not on pancreatic $\alpha$-amylase. Soy isoflavones also decreased glucose transport potency into brush border membrane vesicles or Caco-2 cells. It is concluded that soy isoflavones from hypocotyl, performs hypoglycemic function in GK rats with type 2 diabetes, maybe via suppression of carbohydrate digestion and glucose uptake in small intestine.
\end{abstract}

Key Words: soy isoflavone, hypocotyl, hypoglycemic, diabetes, GK rats

$\mathrm{D}$ iabetes mellitus is a metabolic disorder with hyperglycemia and insulin resistance, and has a considerable impact on life quality and life expectancy. ${ }^{(1)}$ It can be handled by exercise, proper diet and oral antidiabetic agents, and targeted glycemic levels will be achieved. However, these synthetic drugs are expensive and produce some undesirable side effects. Therefore, searching for relatively unexpensive, safe and effective antidiabetic agents is very important, especially for developing countries. ${ }^{(2)}$

Soybean (Glycine max L. Merr.) is known for containing various functional, non-nutrient phytochemicals, such as isoflavones, phytates and saponins. ${ }^{(3)}$ Among them, soy isoflavones are the most commonly noted components. Studies have shown that soy proteins and isoflavones moderate hyperglycemia in diabetic rats ${ }^{(4,5)}$ suggesting a protective effect of soybeans on diabetes mellitus. Report from Ali et al. ${ }^{(6)}$ also revealed that administration with soy isoflavones reduced blood glucose, while blood triglycerides unchanged in diabetic rats. Although these scientific data support the beneficial effect of soy and its isoflavone compo- nents on blood glucose control, the mechanism underlying their preventive effects on diabetes mellitus is still largely unknown.

In recent years, polyphenol-rich functional foods and the bioactive compounds have been developed to be potential preventive agents for diabetes. ${ }^{(7)}$ It is speculated that the diabetic progression could be blocked via the suppression of carbohydrate digestion and absorption in the gastrointestinal tract. ${ }^{(7,8)}$ So, one of the novel oral policies to alleviate hyperglycemia is the inhibition of $\alpha$ amylase and $\alpha$-glucosidase, as well as glucose absorption in the small intestine. ${ }^{(8)}$ In the small intestine, carbohydrates are degraded into polysaccharides and disaccharides by $\alpha$-amylase, then further into monosaccharides by $\alpha$-glucosidase, and finally, absorbed into small intestinal epithelial cells. ${ }^{(8)}$ Our study previously revealed that soy isoflavones are the potential inhibitors of yeast $\alpha$ glucosidase, ${ }^{(9)}$ and Rasouli et al. $^{(7)}$ also speculated that daidzein, one of the soy isoflavones, could inhibit the $\alpha$-glucosidase using molecular docking. To further investigate the preventive role of soy isoflavones from hypocotyl (SIF) on postprandial hyperglycemia, we determined the effect of single and long-term administration of SIF on blood glucose control in Goto-Kakizaki (GK) diabetic rats.

The GK rats, non-obese diabetic animals with low insulin secretion, are the relevant animal model suitable for studying the human type 2 diabetes. ${ }^{(2,10)}$ Since intensive glycemic control is the major goal in treatment of diabetes and essential for reducing the risk of diabetic complications, ${ }^{(11)}$ it is worth studying the effect of soy isoflavones on glycemic control in GK rats with type 2 diabetes. The purpose of the present study was to assess the effect of chronic feeding of SIF on glycemic control in GK diabetic rats and evaluate its possible use as a hypoglycemic agent. We also focused on the hypoglycemic mechanism of SIF in vitro, including the inhibitory potentials on the carbohydrate digestive enzyme activities and glucose uptake in the small intestine.

\section{Materials and Methods}

Chemicals. Test kits for cholesterol, triglyceride and glucose were obtained from Wako Pure Chemical Industries (Osaka, Japan). Lipid peroxide (LPO) test kit was purchased from Kyowa Medex Company (Tokyo, Japan). ELISA kit for insulin were from Wako Pure Chemical Industries; and ELISA kits for glycosylated serum protein (GSP), C-reactive protein (CRP), interleukin (IL)-6, and tumor necrosis factor (TNF)- $\alpha$ were from BD Bioscience (San Jose, CA). Acarbose (ACR) was obtained from Wako Pure Chemical Industries; daidzein, daidzin, malonyldaidzin, glycitein, glycitin, malonylglycitin and malonylgenistin were purchased from Fujicco Company Ltd. (Kobe, Japan);

${ }^{\dagger}$ These authors contributed equally to this work.

*To whom correspondence should be addressed.

E-mail: quanjs@ybu.edu.cn (JQ); yinxz@ybu.edu.cn (XY) 
genistein and genistin were obtained from Extrasynthese (Genay, France).

Preparation and characterization of test extract. Soybean seeds were obtained from the National Institute of Agrobiological Resources (Ibaraki, Japan); Soybean hypocotyl was shaken with $50 \%$ methanol overnight and the solution was filtered, and spraydried. The yield (w/w) of the hypocotyl extract was $14.9 \%$ for the soybean seeds in terms of dry weight. The hypocotyl extract was then dissolved in $10 \%$ methanol and applied to ODS column (YMC, ODS-A60-S150, $5 \mathrm{~cm} \times 74 \mathrm{~cm}$ ). ${ }^{(12)}$ The column was eluted step-wisely with $10 \%, 30 \%$ and $80 \%$ methanol. Each fraction was collected, evaporated to dryness under vacuum and finally lyophilized. ${ }^{(12)}$

To guarantee reproducibility of pharmacological experiments, isoflavone contents of fractions and SIF were analyzed with TLC and HPLC before further application. ${ }^{(12)}$ TLC was performed on a silica gel, and spots were visualized first by fluorescence scanning at $254 \mathrm{~nm}$, then by sulfuric acid spraying and heating. HPLC was carried out using Waters $600 \mathrm{E}$ system as before. ${ }^{(12)}$ The results revealed that $10 \%$ methanol fraction consisted of soybean oligosaccharides; $30 \%$ methanol fraction $\left(\mathrm{F}_{30}\right)$ consisted of malonylated soy isoflavone glycosides; $80 \%$ methanol fraction mostly consisted of soyasaponins and a small amount of isoflavones (non-malonylated isoflavone glycosides and isoflavone aglycones). Therefore, the mixture of $\mathrm{F}_{30}$ was used as precursor of SIF in this experiment. The yield $(\mathrm{w} / \mathrm{w})$ of $\mathrm{F}_{30}$ was $8.4 \%$ in terms of dry weight. The composition was as follows (\%): malonyldaidzin 49.8, malonylglycitin 19.7, malonylgenistin 13.2, daidzin 2.5, glycitin 0.8 , genistin 4.2 , acetyldaidzin 1.1 , acetylglycitin 0.5 (Fig. 1A). Finally, the above-mentioned $\mathrm{F}_{30}$ was hydrolyzed with $6 \mathrm{M}$ hydrochloric acid solution for $4 \mathrm{~h}$. The hydrolysate was extracted with ethyl acetate, distilled and freeze dried to obtain SIF powder. The composition of SIF was as follows (\%): daidzein 61.7, glycitein 21.5 , genistein 16.2 (Fig. 2B).

Single administration test in normal Wistar and GK diabetic rats. Twenty-week-old male GK type 2 diabetic rats were randomly assigned to three groups: the control, ACR (positive control) and SIF treatment groups, each consisting of 10 animals. After overnight fasting, the control group received maltose solution at dose of $2 \mathrm{~g} / \mathrm{kg}$ body weight (BW), whereas the maltose solution $(2 \mathrm{~g} / \mathrm{kg} \mathrm{BW})$ containing SIF $(150 \mathrm{mg} / \mathrm{kg} \mathrm{BW})$ or ACR $(3 \mathrm{mg} / \mathrm{kg} \mathrm{BW})$ was administered to SIF or ACR treatment groups, respectively. The dose of SIF treatment were selected based on our previous report, ${ }^{(12)}$ as well as its efficacy in obese Zucker rats. ${ }^{(5)}$ This dose of SIF is approximately equivalent to $3.5 \mathrm{~g} / \mathrm{kg} \mathrm{BW}$ of hypocotyl extract, and equal to $20 \mathrm{~g} / \mathrm{kg} \mathrm{BW}$ of soybean hypocotyl, in terms of dry weight.

Blood glucose (BG) was determined at 0, 30, 60 and $120 \mathrm{~min}$ after maltose load with a blood glucose test meter (Bayer, Leverkusen, Germany). During the oral maltose tolerance test, the glucose responses were calculated as the corresponding incremental integrated glucose values. Incremental areas under the curve (AUC) of changes in blood glucose were calculated by the following formula: ${ }^{(2)}$

$$
\mathrm{AUC}=\sum\left\{\left[\left(\mathrm{C}_{\mathrm{i}}-\mathrm{C}_{0}\right)+\left(\mathrm{C}_{\mathrm{i}+1}-\mathrm{C}_{0}\right)\right] \times\left(\mathrm{t}_{\mathrm{i}+1}-\mathrm{t}_{\mathrm{i}}\right)\right\} / 2
$$

Long-term administration test in GK diabetic rats. Sixweek-old male GK type 2 diabetic rats were randomly assigned to the control, ACR (positive control) and SIF treatment groups, each consisting of 10 animals. The control group was fed on a standard laboratory chow, while SIF and ACR treatment groups were fed diets containing SIF $(150 \mathrm{mg} / \mathrm{kg} \mathrm{BW})$ or ACR $(3 \mathrm{mg} / \mathrm{kg} \mathrm{BW})$ for a period of 16 weeks. The composition of the standard diet was as follows (\%). Corn starch 30.0, sucrose 25.0, casein 25.0, corn oil 10.0 , cellulose powder 5.0 , mineral mixture 3.5 , vitamin mixture 1.0 , methionine 0.3 and choline bitartrate $0.2 .^{(12)}$ The mixtures of minerals and vitamins were according to AIN-93G MX and AIN-
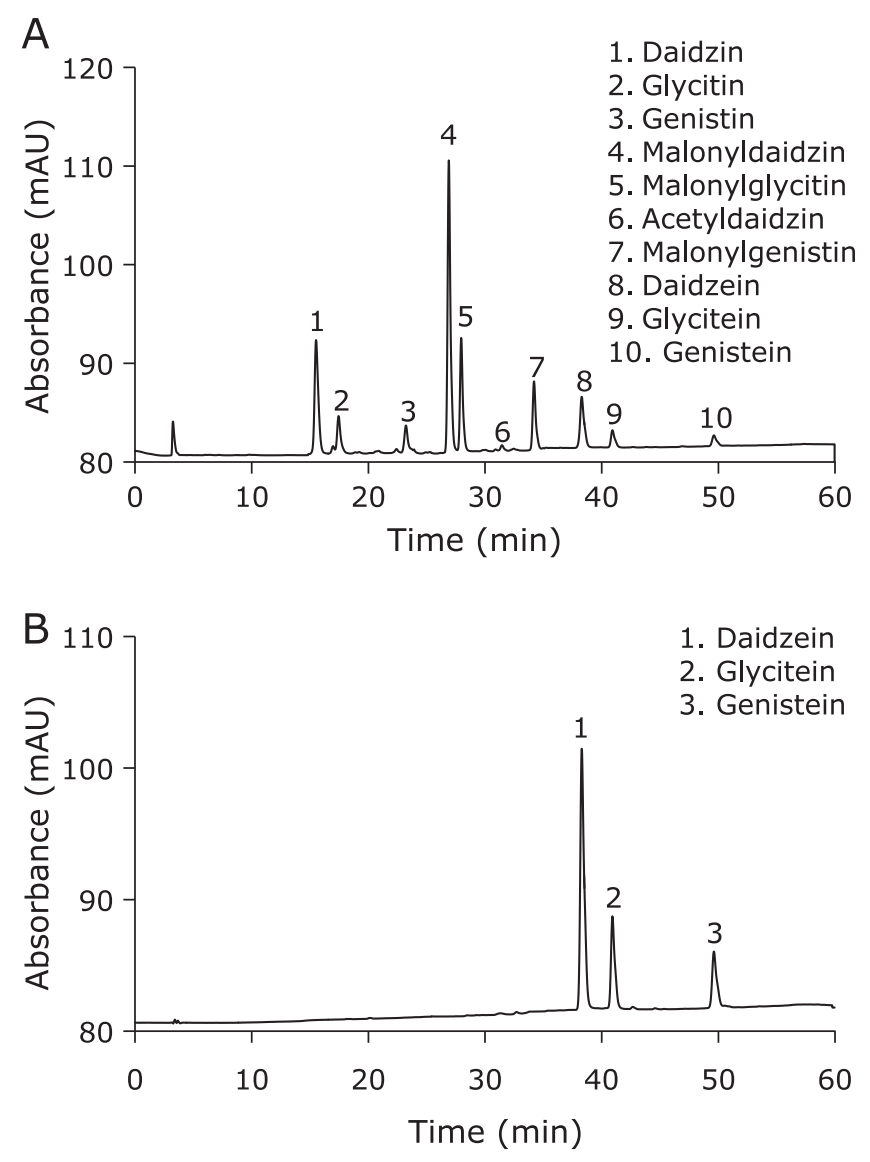

Fig. 1. HPLC chromatograms of $F_{30}(A)$ and SIF (B) showing isoflavone glycoside and aglycone peaks, respectively. HPLC was carried out using Waters 600E system. Column: YMC-packed ODS-AM-303 column $(5 \mu \mathrm{m}$, $4.6 \mathrm{~mm} \times 50 \mathrm{~mm}$ ); UV-detection: $260 \mathrm{~nm}$; mobile phase: a linear gradient of acetonitrile from $15 \%$ to $35 \%$ containing $1 \%$ trifluoroacetic acid.

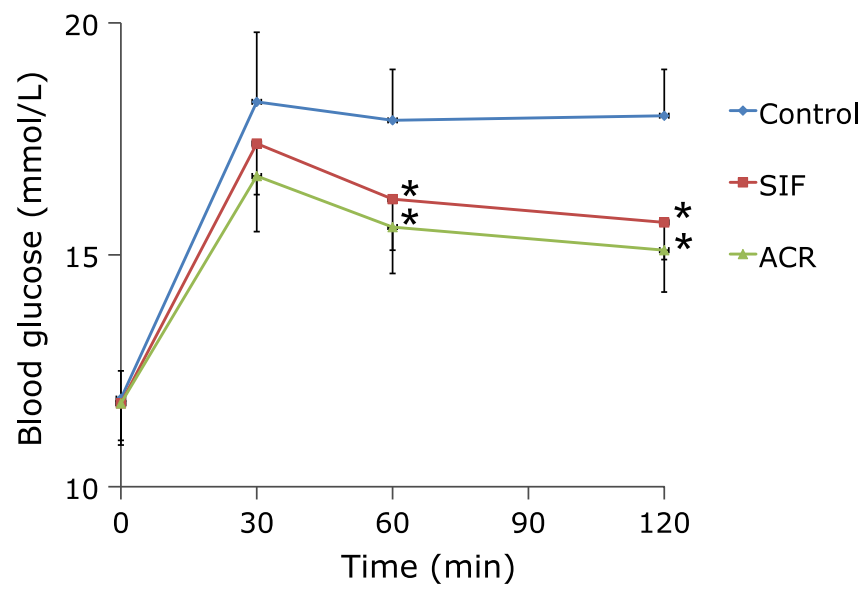

Fig. 2. Effect of single administration of SIF on BG levels in maltoseloaded rats. Maltose ( $2 \mathrm{~g} / \mathrm{kg} \mathrm{BW})$ and SIF (150 mg/kg BW) or ACR $(3 \mathrm{mg} / \mathrm{kg}$ $\mathrm{BW}$ ) were orally administered to the rats. The results are expressed as means \pm SE of ten rats. ${ }^{*} p<0.05$, compared with control group.

93G VX. ${ }^{(13)}$ SIF was added to the diet at the expense of starch. Rats were allowed free access to water and food, and water and food intake were monitered every day. Experimental animals were kept at $24 \pm 1^{\circ} \mathrm{C}$, under a natural light-dark regimen. Experimentations were performed in strict accordance with recommendations of 
Institutional Animal Ethics Committee of Yanbian University Medical College.

Blood samples were collected and tested for fasting BG at 4week intervals. Fasting BG was measured with a blood glucose test meter (Bayer). An oral maltose tolerance test was carried out at week 12 and 16. On test days, animals were fasted overnight, and then received maltose solution at dose of $2 \mathrm{~g} / \mathrm{kg} \mathrm{BW}$. Postpradial $\mathrm{BG}$ was determined at $0,30,60$ and $120 \mathrm{~min}$ after maltose load, and the incremental AUC of BG levels were calculated as before. $^{(2)}$

At week 16, rats were anesthetized, sacrificed, and then blood samples were withdrawn. Blood insulin, GSP, CRP, IL-6, TNF- $\alpha$ and LPO were assayed in accordance with the manufacturer's instructions. Blood lipids and other parameters were detected with a biochemical autoanalyser (Hitachi 736-15, Tokyo, Japan) with respective commercial test kits.

Carbohydrate digestive enzyme inhibitory assay in vitro.

The small intestine of GK rat was dissected and washed out, the mucosa was scraped off and homogenized, and then the supernatant was used as a crude enzyme after centrifugation. ${ }^{(8)}$ The total $\alpha$-glucosidase inhibitory assay was done by the method described by Watanabe et al., ${ }^{(14)}$ and the total activity was measured by monitoring $p$-nitrophenyl released from $p$-nitrophenyl-glucoside at $400 \mathrm{~nm}$. Activities of sucrase or maltase were determined by monitoring glucose liberated from sucrose or maltose at $505 \mathrm{~nm}$ with a Glucose B Test kit (Wako). ${ }^{(8)}$ Porcine pancreatic $\alpha$-amylase suspension (Sigma, type 1-A, St. Louis, MO) was diluted and the activity was determined by monitoring starch at $700 \mathrm{~nm}$ by iodine method. ${ }^{(8)}$

Inhibition of glucose uptake into intestinal brush-border membrane. Brush-border membrane vesicles (BBMVs) were prepared by the $\mathrm{MgCl}_{2}$ precipitation method from rabbit small intestine, ${ }^{(15)}$ and uptake of D-glucose was measured by a rapid filtration technique. ${ }^{(16)}$ The radioactivity was counted in a scintillation counter (LKB Wallac 1209 Rackbeta). Incubation was terminated at a time corresponding to peak glucose uptake. Time for peak glucose uptake varies among BBMV batches from 10 to $20 \mathrm{~s}$.

Inhibition of glucose uptake in intestinal epithelial cells.

Caco-2 cells were obtained from Keygen Biotechnology (Nanjing, China). Cells were grown routinely in DMEM (Biological Industries, Kibbutz Beit-Haemek, Israel) containing $10 \%$ fetal bovine serum (Gemini Bio-Products, West Sacramento, CA), at $37^{\circ} \mathrm{C}$ in $5 \% \mathrm{CO}_{2}$. Cells were grown to $80 \%$ confluence, and seeded at the density of $2 \times 10^{5}$ cells/well on 12 -well cell culture plates. Dglucose uptake was measured according to the previous report. ${ }^{(8)}$ The radioactivity in each monolayer was measured with a liquid scintillation counter (LKB Wallac 1209 Rackbeta, Turku, Finland).

Statistical analysis. Results were presented as mean $\pm \mathrm{SE}$. For the single and long administration experiment, data were analyzed by two-way ANOVA followed by Dunnett's post-hoc test using SPSS 20.0 software (SPSS Inc., Chicago, IL). In the long-term experiment, statistical significances of biochemical parameters were determined by one-way ANOVA followed by Tukey's post-hoc test. Differences were considered as being statistically significant at $p<0.05$.

\section{Results}

Effects of single administration of SIF in GK diabetic rats. Figure 2 and Table 1 represent the results of the single administration test in GK diabetic rats. The BG levels peaked at 30 min after maltose load, and the postprandial BG levels following SIF or ACR treatments were reduced at 60 and 120 min after oral maltose load as compared with the control group (Fig. 2, $p<0.05$ ). These results suggest that SIF and ACR have the maltose intolerance ameliorating effects in GK diabetic rats. For GK diabetic rats, the total AUC was significantly lower in ACR group than the control group (Table $1, p<0.05$ ). The total AUC of SIF group was tended to decrease, though the difference was not statistically significant as compared with the control group (Table $1, p=0.09$ ). No statistically significant differences of BG and total AUC were observed between SIF and ACR groups (Fig. 2, Table 1, $p>0.05$ ).

Effects of long-term administration of SIF in GK diabetic rats.

Assessment of $B W$, food and water intakes of the animals.

Throughout the experimental period, BWs of GK rats continued to increase in all groups, but no significant difference was observed among the rats from the control, SIF and ACR treatment groups (Fig. 3). Neither the water intake (WI) nor food intake (FI) was significantly different among the three groups during the experiment (Fig. 4).

Assessment of fasting $B G$ and oral maltose tolerance. Figure 5 shows the effect of SIF on fasting BG in GK rats. The fasting BGs of all groups showed the tendency to elevate from week 0 to 16 . At week 12, statistically insignificant reductions in fasting BGs were observed in SIF and ACR groups compared with the control group $(p>0.05)$. However, chronic administration with SIF or ACR for a period of 16 weeks produced a significant reduction in fasting $B G$ levels compared with the control GK rats (Fig. 5, $p<0.05)$.

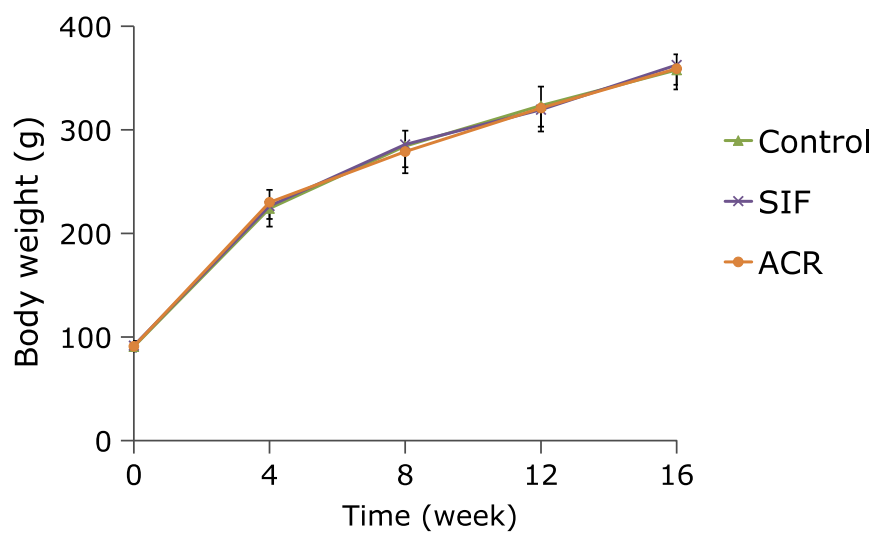

Fig. 3. Effect of SIF on BWs of GK rats in long-term administration test. SIF $(150 \mathrm{mg} / \mathrm{kg} \mathrm{BW})$ or ACR $(3 \mathrm{mg} / \mathrm{kg} \mathrm{BW})$ were orally administered to the rats for 16 weeks. The results are expressed as means \pm SE of ten rats.

Table 1. The incremental AUC of BG levels in single administration test

\begin{tabular}{lccc}
\hline \multirow{2}{*}{$\begin{array}{l}\text { Time period } \\
\text { min) }\end{array}$} & \multicolumn{3}{c}{ Blood glucose AUC (mM-min) } \\
\cline { 2 - 4 } $0-30$ & Control & SIF & ACR \\
$30-60$ & $185.2 \pm 16.5$ & $84.7 \pm 12.3$ & $73.3 \pm 14.3$ \\
$60-120$ & $362.5 \pm 52.4$ & $151.1 \pm 22.4$ & $129.2 \pm 26.4^{*}$ \\
Total & $644.3 \pm 94.3$ & $270.1 \pm 40.3^{*}$ & $218.3 \pm 35.2^{*}$ \\
\hline
\end{tabular}

Maltose $(2 \mathrm{~g} / \mathrm{kg} \mathrm{BW})$ and SIF $(150 \mathrm{mg} / \mathrm{kg} \mathrm{BW})$ or ACR $(3 \mathrm{mg} / \mathrm{kg} \mathrm{BW})$ were orally administered to the rats. The results are expressed as means \pm SE of ten rats. ${ }^{*} p<0.05$, compared with control group. 


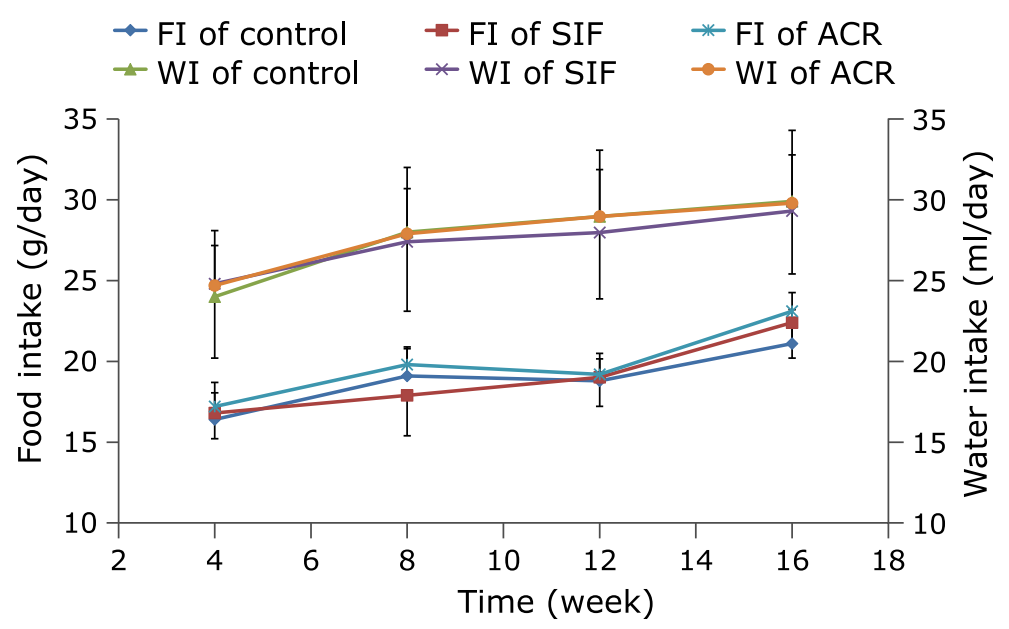

Fig. 4. Effect of SIF on food intake (FI) and water intake (WI) of GK rats in long-term administration test. SIF (150 mg/kg BW) or ACR ( $3 \mathrm{mg} / \mathrm{kg} \mathrm{BW)}$ were orally administered to the rats for a period of 16 weeks. The results are expressed as means \pm SE of ten rats.

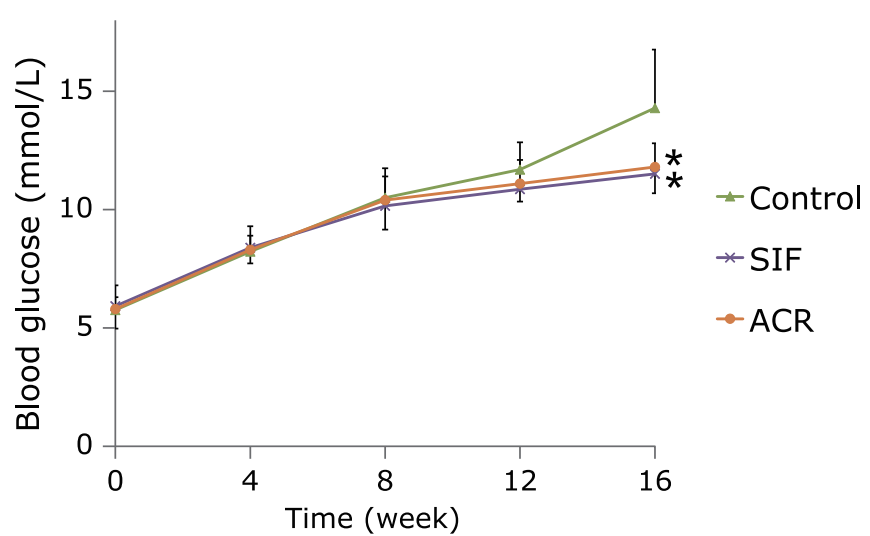

Fig. 5. Effect of SIF on fasting BG levels of GK rats in long-term administration test. SIF $(150 \mathrm{mg} / \mathrm{kg} \mathrm{BW})$ or ACR $(3 \mathrm{mg} / \mathrm{kg} \mathrm{BW})$ were orally administered to the rats for 16 weeks. Blood samples were tested for fasting BG at 4-week intervals. The results are expressed as means $\pm \mathrm{SE}$ of ten rats. ${ }^{*} p<0.05$, compared with control group.

In parallel with the amelioration of fasting BG levels, there were statistically significant reductions in postprandial BG levels in rats after long-term administration of SIF or ACR. Maltose intolerance ameliorating effects of SIF and ACR were apparently observed at week 12 and 16. As shown in Fig. 6, GK rats of the control group showed basal hyperglycemia and the hyperglycemia was aggravated by oral maltose challenge and failed to fall back to fasting BG level after $120 \mathrm{~min}$, indicating impaired maltose tolerance. At week 12, the postprandial BG levels of the SIF and ACR treatment groups decreased significantly at 30 and $60 \mathrm{~min}$ (Fig. 6A, $p<0.05$ ), and total AUCs were tended to decrease, though the differences between the control and SIF or ACR treatment groups were not statistically significant, respectively (Table 2 , $p>0.05$ ). At week 16, the maltose intolerance ameliorating effects of SIF and ACR were more clearly observed. Postprandial BG levels of SIF and ACR treatment groups were significantly lower than those of the control group at all time points checked (Fig. 6B, $p<0.05$ ), but there were no statistically significant differences of total AUCs among the three groups of diabetic rats (Table 2, $p>0.05$ ).

Assessment of insulin, GSP, LPO, CRP, TNF- $\alpha, I L-6$ and other biochemical parameters. In this experiment, GSP level was also studied as an index of glycemic control in GK rats. At the end of the experiment, a small but significant reduction in GSP level was
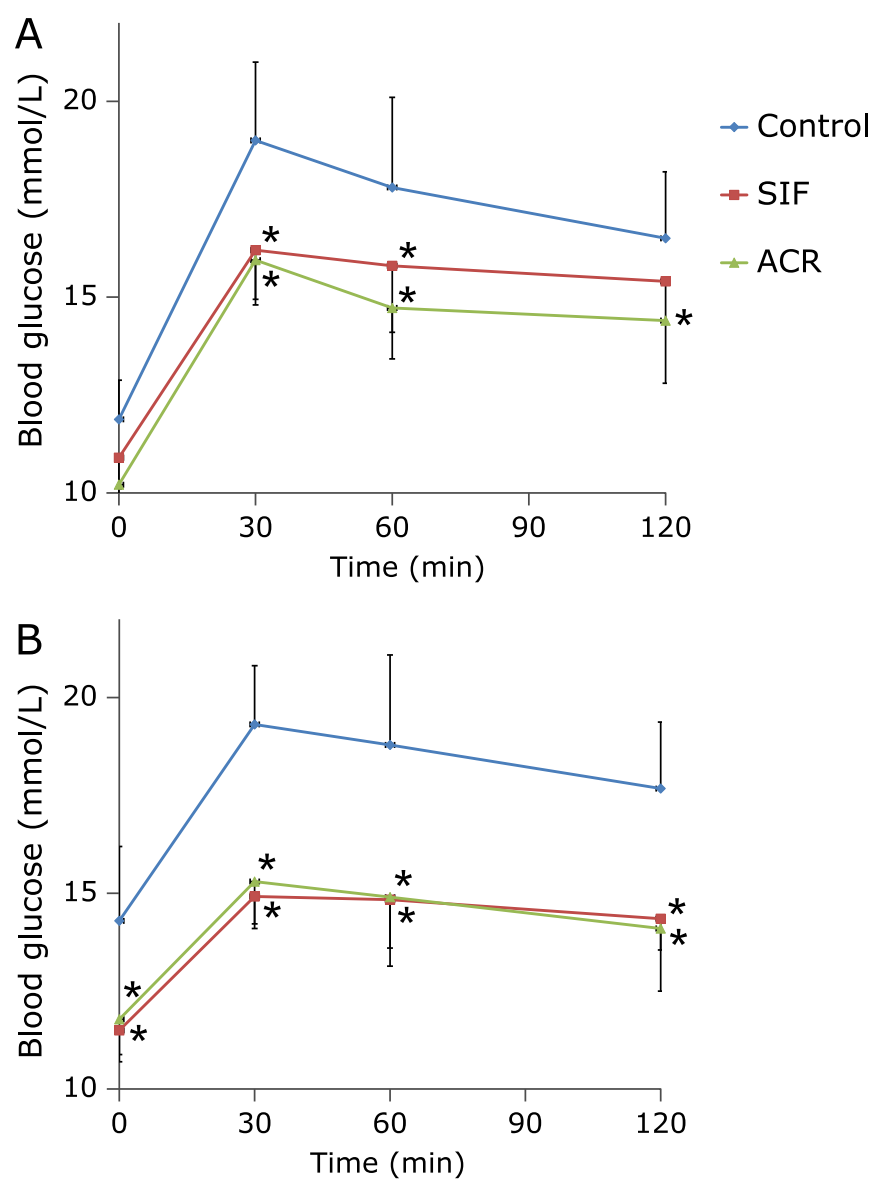

Fig. 6. Effect of long-term administration of SIF on BG levels in maltoseloaded GK rats at week 12 (A) and 16 (B). SIF (150 mg/kg BW) or ACR $(3 \mathrm{mg} / \mathrm{kg} \mathrm{BW})$ were orally administered to the rats for 16 weeks. At week 12 (A) and 16 (B), animals were fasted and an oral maltose tolerance test was carried out. The results are expressed as means \pm SE of ten rats. * $p<0.05$, compared with control group.

observed in GK rats administered with SIF or ACR as compared with the control GK rats (Table 3). However, no significant treatment effect was found for blood insulin of GK rats. In addition to a decrease in GSP level, the chronic SIF administration 
Table 2. The incremental AUC of BG levels of GK rats in long-term administration test

\begin{tabular}{llrrr}
\hline \multirow{2}{*}{ Week } & Time period & \multicolumn{3}{c}{ Blood glucose AUC (mM.min) } \\
\cline { 2 - 5 } & $(\mathrm{min})$ & Control & \multicolumn{1}{c}{ SIF } & \multicolumn{1}{c}{ ACR } \\
\hline 12 & $0-30$ & $106.9 \pm 17.8$ & $79.5 \pm 16.2$ & $86.0 \pm 11.3$ \\
& $30-60$ & $195.7 \pm 32.2$ & $153.4 \pm 24.1$ & $153.7 \pm 19.5$ \\
& $60-120$ & $316.3 \pm 56.1$ & $281.7 \pm 33.6$ & $261.0 \pm 35.4$ \\
& Total & $618.8 \pm 93.9$ & $514.2 \pm 57.4$ & $500.7 \pm 53.5$ \\
16 & $0-30$ & $75.3 \pm 23.6$ & $51.3 \pm 12.4$ & $52.3 \pm 13.3$ \\
& $30-60$ & $142.7 \pm 36.2$ & $101.5 \pm 29.7$ & $99.7 \pm 28.4^{*}$ \\
& $60-120$ & $236.3 \pm 68.5$ & $185.9 \pm 41.3$ & $163.3 \pm 36.2$ \\
& Total & $454.3 \pm 82.7$ & $338.8 \pm 76.3$ & $315.3 \pm 61.5$ \\
\hline
\end{tabular}

SIF (150 mg/kg BW) or ACR ( $3 \mathrm{mg} / \mathrm{kg} \mathrm{BW}$ ) were orally administered to the rats for 16 weeks. At week 12 (A) and 16 (B), animals were fasted and received maltose ( $2 \mathrm{~g} / \mathrm{kg} \mathrm{BW})$, and an oral maltose tolerance test was carried out. The results are expressed as means \pm SE of ten rats. ${ }^{*} p<0.05$, compared with control group.

Table 3. Effect of SIF on blood biochemical parameters of GK rats in long-term administration test

\begin{tabular}{lccc}
\hline Indexes & Control & SIF & ACR \\
\hline Total protein $(\mathrm{g} / \mathrm{dl})$ & $6.2 \pm 0.3$ & $6.1 \pm 0.3$ & $6.3 \pm 0.2$ \\
Triglyceride $(\mathrm{mM})$ & $0.22 \pm 0.03$ & $0.27 \pm 0.04$ & $0.25 \pm 0.03$ \\
Free fatty acid $(\mathrm{mM})$ & $0.73 \pm 0.04$ & $0.69 \pm 0.06$ & $0.62 \pm 0.03$ \\
Cholesterol $(\mathrm{mM})$ & $2.7 \pm 0.5$ & $2.7 \pm 0.6$ & $2.9 \pm 0.4$ \\
HDL-cholesterol $(\mathrm{mM})$ & $1.9 \pm 0.3$ & $1.9 \pm 0.4$ & $2.1 \pm 0.4$ \\
LDL-C $(\mathrm{mM})$ & $0.81 \pm 0.23$ & $0.76 \pm 0.14$ & $0.86 \pm 0.14$ \\
AST $(\mathrm{U} / \mathrm{L})$ & $126 \pm 19$ & $134 \pm 27$ & $130 \pm 26$ \\
ALT $(\mathrm{U} / \mathrm{L})$ & $124 \pm 25$ & $128 \pm 29$ & $122 \pm 23$ \\
Insulin $(\mathrm{mU} / \mathrm{L})$ & $59.4 \pm 18.3$ & $64.4 \pm 18.7$ & $71.9 \pm 20.1$ \\
GSP $(\mathrm{mM})$ & $1.54 \pm 0.17$ & $1.31 \pm 0.19^{*}$ & $1.37 \pm 0.16^{*}$ \\
CRP $(\mathrm{mg} / \mathrm{L})$ & $1.78 \pm 0.23$ & $1.20 \pm 0.24^{*}$ & $1.39 \pm 0.16^{*}$ \\
TNF- $\alpha(\mu \mathrm{g} / \mathrm{L})$ & $81.5 \pm 11.6$ & $75.1 \pm 8.6$ & $70.3 \pm 9.2^{*}$ \\
IL-6 $(\mathrm{ng} / \mathrm{L})$ & $383 \pm 43.1$ & $290 \pm 25.4^{*}$ & $325 \pm 28.5^{*}$ \\
LPO $(\mu \mathrm{M})$ & $11.4 \pm 2.0$ & $9.7 \pm 1.8^{*}$ & $8.1 \pm 0.7^{*}$ \\
\hline
\end{tabular}

SIF (150 mg/kg BW) or ACR (3 mg/kg BW) were orally administered to the rats for 16 weeks. At week 16 , rats were anesthetized, sacrificed, and then blood samples were tested for the biochemical parameters. The results are expressed as means \pm SE of ten rats. ${ }^{*} p<0.05$, compared with control group.

Table 4. $I C_{50}$ values of SIF on rat $\alpha$-glucosidase in vitro

\begin{tabular}{lccc}
\hline \multirow{3}{*}{ Component } & \multicolumn{3}{c}{$\mathrm{IC}_{50}(\mathrm{~g} / \mathrm{L})$} \\
\cline { 2 - 4 } & $\begin{array}{c}\text { Total } \alpha \text {-glucosidase } \\
\left(K_{\mathrm{m}}=0.7 \mathrm{mM}\right)\end{array}$ & $\begin{array}{c}\text { Maltase } \\
\left(K_{\mathrm{m}}=1.7 \mathrm{mM}\right)\end{array}$ & $\begin{array}{c}\text { Sucrase } \\
\left(K_{\mathrm{m}}=21.2 \mathrm{mM}\right)\end{array}$ \\
\hline $\mathrm{F}_{30}$ & $>10$ & 9.4 & 8.8 \\
SIF & 3.1 & 5.6 & 8.6 \\
Daidzein & 2.52 & 4.7 & 8.5 \\
Genistein & 2.39 & 3.9 & 9.4 \\
Glycitein & 8.8 & 8 & $>10$ \\
ACR & 0.12 & 0.19 & 0.34 \\
\hline
\end{tabular}

$K_{\mathrm{m}}$ values were obtained from Lineweaver-Burk plots of rat $\alpha$-glucosidase. The results represent the means of four experiments.

also induced a substantial modification in oxidative and inflammatory damages to the plasma. Compared with the control group, SIF or ACR treatment significantly decreased blood LPO, CRP, TNF- $\alpha$ and IL- 6 levels (Table $3, p<0.05$ ). The levels of blood total protein, triglyceride, cholesterol, HDL-cholesterol and other biochemical parameters, including AST, ALT, were not modified with SIF or ACR treatment, however (Table 3).

Effect of SIF on carbohydrate digestive enzymes and glucose uptake in vitro. Inhibitory potentials of SIF against $\alpha$-glucosidase and $\alpha$-amylase were summarized in Table 4 . SIF exhibited inhibitory activities on total $\alpha$-glucosidase, including maltase and sucrase from rat small intestine. The inhibitory effects of isoflavone aglycones, the major components of SIF, and ACR were also shown in Table 4. However, the inhibitory activities of SIF on pig pancreatic $\alpha$-amylase was so weak, with the inhibitory activities of 10 to $20 \%$ at the concentration of $1 \mathrm{~g} / \mathrm{L}$, that the half inhibitory concentration $\left(\mathrm{IC}_{50}\right)$ values were not able to be detected in this experiment (Data were not shown).

In addition, SIF suppressed concentration-dependently glucose uptake into BBMVs or Caco- 2 cells over the range of $5 \mathrm{~g} / \mathrm{L}$ to $200 \mathrm{~g} / \mathrm{L}$, with the $\mathrm{IC}_{50}$ values of 49 and $12 \mathrm{~g} / \mathrm{L}$, respectively (Fig. 7). $\mathrm{IC}_{50}$ values of phlorizin, a glucose uptake inhibitor, were 1.6 and $0.48 \mathrm{~g} / \mathrm{L}$ in these in vitro systems. 


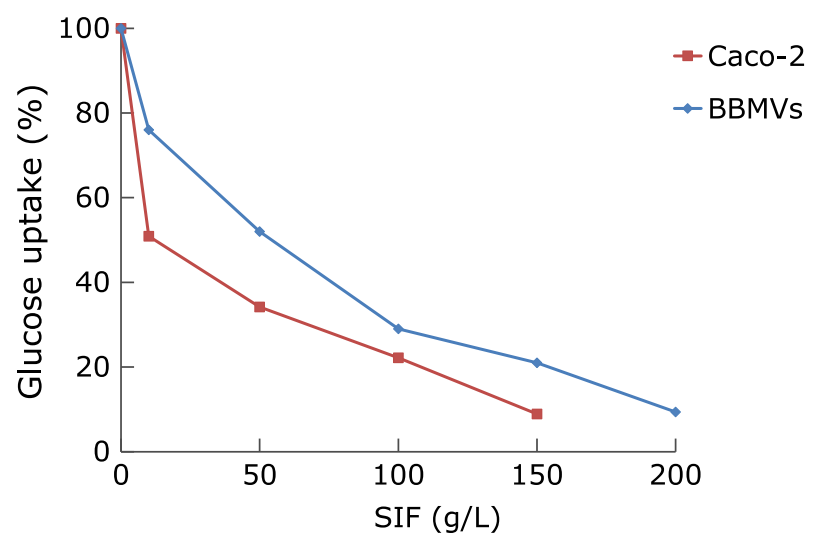

Fig. 7. Effect of SIF on glucose uptake using BBMVs or Caco-2 cells in vitro. The results represent the means of six experiments.

\section{Discussion}

Recent studies have focused on soy phytochemicals, and isoflavones are the most commonly noted bioactive components among them. ${ }^{(3)}$ Soy isoflavones are mostly concentrated in soybean hypocotyls. They occur as glucose-conjugated forms and are hydrolyzed to absorbable aglycones once ingested. ${ }^{(17)}$ The bioavailability of aglycones and glucosides of soy isoflavones is controversial. ${ }^{(18,19)}$ However, it is commonly assumed that isoflavone aglycones are absorbed more quickly and in greater amounts than their glucoside forms. ${ }^{(19)}$ Our previous study indicated that soy isoflavone aglycones had an inhibitory activity against $\alpha$ glucosidase, ${ }^{(9)}$ and that provides a strong rationale for further animal studies and encourages us to investigate the hypoglycemic effect of SIF in GK rats.

In the single administration experiment, we studied whether SIF would suppress the postprandial BG levels after oral maltose load. The results showed that the administration of SIF significantly suppressed the postprandial BG levels at $120 \mathrm{~min}$ after maltose load in GK diabetic rats, whereas only the $\mathrm{AUC}_{60-120 \text { min }}$ of diabetic rats exhibited a significant reduction than the control group. The results of the single administration experiment confirmed the preventive role of SIF on hyperglycemia in postprandial state.

Since a single administration of SIF had a beneficial effect on blood glucose control in diabetes, we further investigated the long-term administration experiment in GK diabetic rats. In this study, chronic administration of SIF induced significant reduction in fasting BG levels and amelioration in oral maltose tolerance in GK diabetic rats. After 12 weeks of treatment with SIF, a maltose intolerance ameliorating effect of SIF was observed, though fasting BG lowering effect was not observed. Significant difference in fasting BG levels between the two groups was noted at week 16 after SIF feeding.

The animal dosage of SIF in this study could be converted to human equivalent dose of $24 \mathrm{mg} / \mathrm{kg} \mathrm{BW}$ based on body surface area, ${ }^{(20)}$ and this amount is far in excess of usual dietary intakes of $20 \sim 30 \mathrm{mg}$ /day isoflavone aglycones among Japanese or Korean populations. ${ }^{(21,22)}$ However, the advantage of ingesting high quantities of soy isoflavones in humans is questionable, given a curvilinear relationship between bioavailability and intake. ${ }^{(23)}$ Considering the decreasing bioavailability with increasing isoflavone intake, the beneficial effect of SIF on fasting blood glucose control are more likely to be achieved by multiple intakes of soy isoflavones rather than from a single highly enriched product, on the basis of the pharmacokinetics. ${ }^{(23)}$ So, an increased clinical efficacy in dietary intervention studies would be expected from a modest intake of soy isoflavones consumed throughout the day, without the potential for long-term negative effects.

We also investigated the effects of SIF on chronic glycaemic status in GK rats. Fasting or postprandial BG reflects only an instant glucose level, while GSP provides a time-averaged glucose level over 1 2 weeks, and may be a valuable parameter for the evaluation of the adequacy of diabetic BG control. ${ }^{(24)}$ During the experimental period, the GSP of SIF-treated diabetic rats decreased significantly as compared to the control rats. This result indicates that chronic glycaemic status in GK diabetic rats were controlled by SIF treatment and this was consistent with the suppression of fasting and postprandial BG levels as described above.

However, this hypoglycemic effect of SIF was not associated with $\mathrm{BW}$, food consumption and water intake, suggesting that SIF has an anti-hyperglycemic potential without affecting energy metabolism. No difference in blood insulin level was either observed between the two groups of GK rats. GK type 2 diabetic rats continue an experimental diabetes model of lack of insulin due to impaired glucose recognition by pancreatic $\beta$-cells. ${ }^{(25)}$ In this study, SIF did not stimulate insulin secretion of GK rats. These results suggest that SIF has the ability to lower BG without the help of endogenous insulin, and the suppression of the BG levels by SIF may be mediated through other pathways.

The major goal of diabetic treatment is to optimize glycemic control to prevent the diabetes-associated complications caused by hyperglycemia. ${ }^{(11)}$ Under hyerglycemic conditions, reactive oxygen species (ROS) are produced via glucose auto-oxidation and no-enzymatic protein glycation in tissues. ${ }^{(26)}$ Type 2 diabetes mellitus is also associated with innate immune activation, and exhibit increased TNF- $\alpha$, IL-1 $\beta$, IL-6 and CRP following IL-6 secretion. ${ }^{(26)}$ Therefore, phytochemicals for treatment of diabetes should possess both the anti-oxidative and anti-inflammatory effects. (27) Beyond an improvement of glycemic control, the chronic SIF administration also induced a substantial modification in indexes of oxidative damage and inflammation to the blood. A significant decrease was apparent for blood LPO, CRP, TNF- $\alpha$ and IL- 6 levels while there was no significant change of other blood biochemical parameters such as total protein, cholesterol, triglyceride, AST and ALT in SIF group compared with the control group. These results partly agree with the findings of Watanabe et al., ${ }^{(28)}$ who have reported soybean hypocotyl tea had an antioxidant activity in humans.

A surge in BG is due to hydrolysis of dietary starch by digestive enzymes such as $\alpha$-glucosidase and $\alpha$-amylase, as well as glucose uptake by the intestinal tract. ${ }^{(29,30)}$ Polyphenol compounds have been proposed to be potential inhibitors of $\alpha$-amylase and $\alpha$ glucosidase, and inhibition of these enzymes can regulate carbohydrate metabolism. ${ }^{(7)}$ To investigate the hypoglycemic mechanism of SIF, we examined the inhibitory effects on carbohydrate digestive enzymes in vitro. Our data showed that SIF had inhibitory effects on $\alpha$-glucosidase, including maltase and sucrase, which are present in the small intestinal mucosa, even though their inhibitory effects on these enzymes were weaker than those of acarbose. However, SIF displayed a much weaker inhibition potential towards pig pancreatic $\alpha$-amylase. These results are in good agreement with the virtual screening studies of Rasouli et al., ${ }^{(7)}$ who speculate that daidzein could inhibit the $\alpha$-glucosidase but not $\alpha$-amylase using molecular docking and virtual screening studies. Inhibition of these carbohydrate digestive enzymes can inhibit intestinal digestion of starch and suppress the BG levels in postprandial state, and could be used without the risk of hypoglycemia. ${ }^{(30)}$

However, inhibition of these carbohydrate digestive enzymes could not prevent glucose uptake in the digestive tract when foodstuffs contain glucose as sugar. So, it might be essential to block the intestinal glucose uptake to suppress the postprandial hyperglycemia. Glucose uptake in the small intestine is mediated by sodium-dependent glucose transporters (SGLTs), and any glucose 
in the lumen is rapidly taken up by SGLTs. ${ }^{(8)}$ To explore the possible influence of SIF on glucose absorption in intestinal tract, we examined glucose uptake by BBMVs or Caco-2 cells after treating with SIF. Phlorizin, a competitive inhibitor of SGLTs, ${ }^{(8)}$ was used as the positive control in this study. Phlorizin is used as a potential pharmaceutical treatment for type 2 diabetes mellitus, and also found to exist in black soybean, recently. ${ }^{(31)} \mathrm{We}$ found that SIF suppressed concentration-dependently glucose uptake into BBMVs or Caco-2 cells, even though its inhibitory effect on glucose uptake was much weaker than that of phlorizin. Although these values are quite higher than the concentrations for digestive enzyme inhibition, they may yet be relevant in vivo since levels of these components in intestinal tract will be much higher than those in blood. . $^{(32)}$

In conclusion, SIF has a preventive effect against hypergly-

\section{References}

1 Lim KH, Han JH, Lee JY, et al. Assessment of antidiabetogenic potential of fermented soybean extracts in streptozotocin-induced diabetic rat. Food Chem Toxicol 2012; 50: 3941-3948.

2 Ndong M, Uehara M, Katsumata S, Suzuki K. Effects of oral administration of Moringa oleifera Lam on glucose tolerance in Goto-Kakizaki and Wistar rats. J Clin Biochem Nutr 2007; 40: 229-233.

3 Ishii Y, Tanizawa H. Effects of soyasaponins on lipid peroxidation through the secretion of thyroid hormones. Biol Pharm Bull 2006; 29: 1759-1763.

4 Lavigne C, Marette A, Jacques H. Cod and soy proteins compared with casein improve glucose tolerance and insulin sensitivity in rats. Am J Physiol Endocrinol Metab 2000; 278: E491-E500.

5 Mezei O, Banz WJ, Steger RW, Peluso MR, Winters TA, Shay N. Soy isoflavones exert antidiabetic and hypolipidemic effects through the PPAR pathways in obese Zucker rats and murine RAW 264.7 cells. J Nutr 2003; 133: 1238-1243.

6 Ali AA, Velasquez MT, Hansen CT, Mohamed AI, Bhathema SJ. Modulation of carbohydrate metabolism and peptide hormones by soybean isoflavones and probiotics in obesity and diabetes. J Nutr Biochem 2005; 16: 693-699.

7 Rasouli H, Hosseini-Ghazvini SM, Adibi H, et al. Differential $\alpha$-amylase/ $\alpha$-glucosidase inhibitory activities of plant-derived phenolic compounds: a virtual screening perspective for the treatment of obesity and diabetes. Food Funct 2017; 8: 1942-1954.

8 Satoh T, Igarashi M, Yamada S, Takahashi N, Watanabe K. Inhibitory effect of black tea and its combination with acarbose on small intestinal $\alpha$-glucosidase activity. J Ethnopharmacol 2015; 161: 147-155.

9 Quan J, Yin X, Kanazawa T, et al. Inhibition of alpha-glucosidase and alphaamylase by soybean isoflavonoids. J Med Sci Yanbian Univ 2001; 24: 243244.

10 Goda T, Suruga K, Komori A, et al. Effects of miglitol, an alpha-glucosidase inhibitor, on glycaemic status and histopathological changes in islets in nonobese, non-insulin-dependent diabetic Goto-Kakizaki rats. Br J Nutr 2007; 98: $702-710$.

11 Roghani M, Vaez Mahdavi MR, Jalali-Nadoushan MR, et al. Chronic administration of daidzein, a soybean isoflavone, improves endothelial dysfunction and attenuates oxidative stress in streptozotocin-induced diabetic rats. Phytother Res 2013; 27: 112-117.

12 Quan J, Yin X, Kanazawa T. Effect of soybean hypocotyl extract on lipid peroxidation in GK rats. J Clin Biochem Nutr 2009; 44: 212-217.

13 Reeves PG, Nielsen FH, Fahey GC Jr. AIN-93 purified diets for laboratory rodents: final report of the American Institute of Nutrition ad hoc writing committee on the reformulation of the AIN-76A rodent diet. J Nutr 1993; 123: $1939-1951$.

14 Watanabe J, Kawabata J, Kurihara H, Niki R. Isolation and identification of alpha-glucosidase inhibitors from tochu-cha (Eucommia ulmoides). Biosci Biotechnol Biochem 1997; 61: 177-178.

15 Vázquez CM, Rovira N, Ruiz-Gutiérrez V, Planas JM. Developmental changes in glucose transport, lipid composition, and fluidity of jejunal BBM. Am J Physiol 1997; 273 (3 Pt 2): R1086-R1093.

16 Garriga C, Moretó M, Planas JM. Hexose transport in the apical and basolateral membranes of enterocytes in chickens adapted to high and low cemia in GK rats with type 2 diabetes mellitus, which may be related to suppression of carbohydrate digestion and glucose uptake in the small intestine. These findings demonstrate that soybean and soy isoflavones are potential candidates for medical food or pharmaceutical supplements in treatment of type 2 diabetes mellitus.

\section{Acknowledgments}

This study was supported by grants from the National Natural Science Foundation of China $(81160539 ; 81360651)$.

\section{Conflict of Interest}

No conflict of interest were disclosed.
$\mathrm{NaCl}$ intakes. J Physiol 1999; 514 (Pt 1): 189-199.

17 Setchell KD, Brown NM, Zimmer-Nechemias L, et al. Evidence for lack of absorption of soy isoflavone glycosides in humans, supporting the crucial role of intestinal metabolism for bioavailability. Am J Clin Nutr 2002; 76: 447453.

18 Kano M, Takayanagi T, Harada K, Sawada S, Ishikawa F. Bioavailability of isoflavones after ingestion of soy beverages in healthy adults. J Nutr 2006; 136: 2291-2296.

19 Zubik L, Meydani M. Bioavailability of soybean isoflavones from aglycone and glucoside forms in American women. Am J Clin Nutr 2003; 77: 14591465.

20 U.S. Department of Health and Human Services, Food and Drug Administration, Center for Drug Evaluation and Research (CDER). Guidance for Industry Estimating the Maximum Safe Starting Dose in Initial Clinical Trials for Therapeutics in Adult Healthy Volunteers. 2005.

21 Surh J, Kim MJ, Koh E, Kim YK, Kwon H. Estimated intakes of isoflavones and coumestrol in Korean population. Int J Food Sci Nutr 2006; 57: 325-344.

22 Wakai K, Egami I, Kato K, et al. Dietary intake and sources of isoflavones among Japanese. Nutr Cancer 1999; 33: 139-145.

23 Stchell KD, Brown NM, Desai PB, et al. Bioavailability, disposition, and dose-response effects of soy isoflavones when consumed by healthy women at physiologically typical dietary intakes. J Nutr 2003; 133: 1027-1035.

24 Hong H, Jai Maeng W. Effects of malted barley extract and banaba extract on blood glucose levels in genetically diabetic mice. J Med Food 2004; 7: 487490.

25 Alvarez C, Bailbe D, Picarel-Blanchot F, Bertin E, Pascual-Leone AM, Portha B. Effect of early dietary restriction on insulin action and secretion in the GK rat, a spontaneous model of NIDDM. Am J Physiol Endocrinol Metab 2000; 278: E1097-E1103.

26 Aslan M, Orhan DD, Orhan N, Sezik E, Yoșilada E. A study of antidiabetic and antioxidant effects of Helichrysum graveolens capitulums in streptozotocininduced diabetic rats. $J$ Med Food 2007; 10: 396-400.

27 Ehses JA, Lacraz G, Giroix MH, et al. IL-1 antagonism reduces hyperglycemia and tissue inflammation in the type 2 diabetic GK rat. Proc Natl Acad Sci U S A 2009; 106: 13998-14003.

28 Watanabe S, Haba R, Terashima K, et al. Antioxidant activity of soya hypocotyl tea in humans. Biofactors 2000; 12: 227-232.

29 Gopal SS, Lakshmi MJ, Sharavana G, Sathalah G, Sreerama YN, Baskaran V. Lactucaxanthin - a potential anti-diabetic carotenoid from lettuce (Lactuca sativa) inhibits $\alpha$-amylase and $\alpha$-glucosidase activity in vitro and in diabetic rats. Food Funct 2017; 8: 1124-1131.

30 Kodama T, Miyazaki T, Kitamura I, et al. Effects of single and long-term administration of wheat albumin on blood glucose control: randomized controlled clinical trials. Eur J Clin Nutr 2005; 59: 384-392.

31 Lee CC, Dudonné S, Dubé P, et al. Comprehensive phenolic composition analysis and evaluation of Yak-Kong soybean (Glycine max) for the prevention of atherosclerosis. Food Chem 2017; 234: 486-493.

32 Vedavanam K, Srijayanta S, O'Reilly J, Paman A, Wiseman H. Antioxidant action and potential antidiabetic properties of an isoflavonoid-containing soyabean phytochemical extract (SPE). Phytother Res 1999; 13: 601-608. 